\title{
ESTIMATION OF CHLOROPHYLL FLUORESCENCE UNDER NATURAL ILLUMINATION FROM HYPERSPECTRAL DATA
}

\author{
Pablo J. ZARCO-TEJADA*, John R. MILLER ${ }^{* *}$, Gina H. MOHAMMED ${ }^{* * *}$, Thomas L. NOLAND ${ }^{* * *}$, and Paul \\ H. SAMPSON*** \\ ${ }^{*}$ Centre for Research in Earth and Space Science (CRESS), York University, Toronto, Canada \\ pzarco@yorku.ca \\ ** Dept. of Physics and Astronomy, York University \& Centre for Research in Earth and Space Technology \\ (CRESTech), Toronto, Canada \\ jrmiller@yorku.ca \\ ${ }^{* * *}$ Ontario Forest Research Institute (OFRI), Ontario Ministry of Natural Resources, Sault Ste. Marie, Ontario, Canada \\ gina.mohammed@mnr.gov.on.ca
}

KEY WORDS: Reflectance, Chlorophyll Fluorescence, Red Edge, Radiative Transfer, CASI, Hyperspectral

\begin{abstract}
This paper reports a series of laboratory and field measurements of spectral reflectance under artificial and natural light conditions which demonstrate that effects of natural chlorophyll fluorescence are observable in the reflectance red edge spectral region. These are results from the progress made to link physiologically-based indicators to optical indices from hyperspectral remote sensing in the Bioindicators of Forest Sustainability Project. This study is carried out on twelve sites of Acer saccharum M. in the Algoma Region, Ontario (Canada), where field measurements, laboratory-simulation experiments, and hyperspectral CASI imagery have been carried out in 1997, 1998, 1999 and 2000 campaigns. Leaf samples from the study sites have been used for reflectance and transmittance measurements with the Li-Cor Model 1800 integrating sphere apparatus coupled to an Ocean Optics Model ST1000 fibre spectrometer in which the same leaves are illuminated alternatively with and without fluorescence-exciting radiation. A study of the diurnal change in leaf reflectance spectra, combined with fluorescence measurements with the PAM-2000 Fluorometer show that the difference spectra are consistent with observed diurnal changes in steady-state fluorescence. Small canopies of Acer saccharum M. have been used for laboratory measurements with the CASI hyperspectral sensor, and under natural light conditions with a fibre spectrometer in diurnal trials, in which the variation of measured reflectance is shown experimentally to be consistent with a fluorescence signature imposed on the inherent leaf reflectance signature. Such reflectance changes due to $\mathrm{CF}$ are measurable under natural illumination conditions, although airborne experiments with the CASI hyperspectral sensor produced promising but less convincing results in two diurnal experiments carried out in 1999 and 2000, where small variations of reflectance due to the effect of CF were observed.
\end{abstract}

\section{INTRODUCTION}

The objective of the Bioindicators of Forest Sustainability Project (Mohammed et al., 1997; Sampson et al., 1998) is to develop links between physiologically-based bio-indicators from field and laboratory data and optical indices from hyperspectral remote sensing data for assessing forest condition. Previous work (Zarco-Tejada et al., 1999a; 1999b) showed that optical indices calculated from single leaf reflectance data, infinite reflectance models from optically-thick simulation formulae, and canopy reflectance models, progressively more closely represented the observed abovecanopy reflectance spectra. Optical indices calculated from modelled canopy reflectance through infinite and canopy reflectance models were shown to be able to be used for estimation of pigment content over closed deciduous canopies of Acer saccharum M, and showed high correlations with ground truth chlorophyll fluorescence (CF). The strong correlation relationships obtained between selected optical indices calculated in the 690 and $750 \mathrm{~nm}$ spectral region from airborne CASI hyperspectral data with ground measured CF were studied in detail in Zarco-Tejada et al. (in press, a, b). Experiments were carried out at different levels of study in the laboratory and through the development of a radiative transfer model (FRT) that simulates the effect of the fluorescence signal superimposed to the reflectance spectrum. This paper reports on further research carried out in this research theme, in which experiments with a fibre spectrometer were carried out using small canopies of seedlings under natural illumination conditions, and with CASIairborne campaigns over Acer saccharum M. study sites in 1999 and 2000 with specific bandsets to investigate the ability to detect CF from an airborne sensor. 


\section{LEVELS OF STUDY}

The effects of the CF signature on vegetation apparent reflectance were studied in a series of experiments in 1997 , 1998, 1999 and 2000 at different levels of scale. Four different levels of study were carried out, from the leaf level to the canopy, in order to investigate whether the effects of CF on apparent reflectance are measurable in spite of increasing complexity: i) at leaf level, with data collected from Acer saccharum M. study sites to develop relationships between leaf reflectance and CF; diurnal studies were also carried out at leaf level to study the effects of changes in leaf apparent reflectance due to diurnal CF patterns; ii) at canopy simulation level in the laboratory using the CASI hyperspectral sensor and maple seedlings; iii) at canopy simulation level with a fibre spectrometer in a diurnal trial using Acer saccharum M. seedlings under natural illumination conditions; and iv) at above canopy over selected forest sites with the airborne CASI hyperspectral sensor in a series of diurnal experiments with different CASI modes of operation and bandsets.

\section{EXPERIMENTAL METHODS AND DATA COLLECTION}

Leaf material used for experimental studies reported in this paper was sampled from Acer saccharum M. potted trees grown in the greenhouse, and from twelve 30x30m Acer saccharum M. study sites in the Algoma Region, Canada, where a total of 440 single leaf samples were collected per campaign for biochemical analysis and measurement of chlorophyll fluorescence, leaf chlorophyll and carotenoid content. Experimental methods to measure leaf and canopy reflectance were carried out at the different levels of study, and are described in the following sections. Chlorophyll fluorescence was analyzed with a Pulse Amplitude Modulation (PAM-2000) Fluorometer (Heinz Walz GmbH, Effeltrich, Germany), an instrument that has been used widely in basic and applied fluorescence research (Mohammed et al., 1995). Procedures used for measuring Fv/Fm and effective quantum yield $\Delta \mathrm{F} / \mathrm{Fm}$ ' were based on standard methodologies as documented in the PAM-2000 manual (Heinz-Walz-GmbH, 1993). For measurement of maximal fluorescence induction $\mathrm{Fv} / \mathrm{Fm}$, leaves were dark-adapted in bags at room temperature for at least $30 \mathrm{~min}$.

\subsection{Leaf-level measurements of reflectance and transmittance}

Measurements of reflectance and transmittance at leaf level were acquired on leaf samples using a Li-Cor 1800-12 Integrating Sphere apparatus coupled by a $200 \mu \mathrm{m}$ diameter single mode fibre to an Ocean Optics model ST 1000 spectrometer, with a 1024 element detector array, $0.5 \mathrm{~nm}$ sampling interval and $\sim 7.3 \mathrm{~nm}$ spectral resolution in the 340-860 nm range. A modification was made to the standard Li-Cor 1800-12 Integrating Sphere apparatus involving a second Li-Cor Lamp/Collimator housing and the insertion of a Schott RG 695 coloured long-pass glass filter blocking radiant flux at $\lambda<695 \mathrm{~nm}$ at the exit aperture of one of the illuminator units (Zarco-Tejada et al., in press, a). These two light sources enabled measurements alternately of reflectance and transmittance of a given sample without fluorescence and including the effect of fluorescence. Diurnal and time-decay studies were carried out with leaf samples in order to study variations in the apparent leaf reflectance and transmittance due to normal diurnal changes of chlorophyll fluorescence, and to the effects of fluorescence time-decay on the measurements of apparent spectral reflectance, respectively (Zarco-Tejada et al., in press, a).

\subsection{Canopy reflectance in laboratory and under natural illumination}

CASI hyperspectral canopy reflectance measurements in the laboratory were made from a small canopy of Acer saccharum M. seedlings using a Bi-Directional Reflectance Factor (BRF) facility. The CASI sensor was placed at height of $2.5 \mathrm{~m}$ above the canopy of plant material, and operated in a hyperspectral mode at maximum spectral resolution with 288 channels, spectral spacing of $1.8 \mathrm{~nm}$ and nominal bandwidth of $2.5 \mathrm{~nm}$. Collimated illumination at $45^{\circ}$ inclination was provided by a regulated $1000 \mathrm{~W}$ halogen light source and a collimating lens. The raw 12 bit CASI data were calibrated to spectral radiance and a Spectralon reflectance panel placed on the moveable platform with the plant material enabled reflectance calculation. A filter holder was custom-designed to permit a Schott RG695 high pass filter to be placed in front of the $1000 \mathrm{~W}$ halogen light source in order to restrict incident radiant energy on the scene to $\lambda>705 \mathrm{~nm}$. This facilitated the collection of canopy reflectance measurements with CASI in the absence of fluorescence generating radiation, similar to measurement protocols at the leaf level.

A canopy of Acer saccharum M. seedlings was used for measurements of canopy reflectance under natural illumination conditions using up/down radiance/irradiance optical head coupled by two $200 \mu \mathrm{m}$ diameter fibre to the same Ocean Optics model ST 1000 triple spectrometer described previously, with $0.5 \mathrm{~nm}$ sampling interval, $7.3 \mathrm{~nm}$ spectral resolution, and 340-860 $\mathrm{nm}$ range. The radiometer optics with the fibre spectrometer detector was directed at nadir view over the seedling canopy using a $1.5 \mathrm{~m}$ tripod, and measurements were made at $8.20 \mathrm{~h}, 8.42 \mathrm{~h}, 9.02 \mathrm{~h}, 10.47 \mathrm{~h}, 11.34 \mathrm{~h}$, $12.52 \mathrm{~h}$, and $13.37 \mathrm{~h}$ to capture variations in apparent reflectance due to the effect of diurnal changes in $\mathrm{CF}$. 


\subsection{Field-airborne canopy reflectance measurements under natural illumination with CASI hyperspectral sensor}

Airborne CASI data were collected over twelve sites of Acer saccharum M. in 1997, 1998, 1999 and 2000. Mean reflectance values per plot were calculated from the imagery in each Acer saccharum M. study site of 20x20 m. CASI data were acquired in the hyperspectral reflectance mode, with $2 \mathrm{~m}$ spatial resolution and 72 spectral channels $(7.5 \mathrm{~nm}$ spectral bandwidth). A CASI diurnal mission was carried out in July 1999 collecting data over two study sites at different times of the day, 8.00h, 9.30h, 12.20, and 16.12h along with ground truth CF measurements with PAM-2000. A second diurnal experiment over two sites was carried out in June 2000 with a specific CASI mode of operation in order to allow for higher spatial resolution data with spectral bands centered at the PSII photosystem. CASI data were collected in 9 spectral bands at 680.47, 684.26, 688.06, 691.86, 695.66, 699.46, 703.26, 707.06, and $710.87 \mathrm{~nm}$ and $0.56 \times 1.08 \mathrm{~m}$ spatial resolution, re-sampled to $0.5 \times 0.5 \mathrm{~m}$. Radiometric calibration and atmospheric correction of the CASI data was performed as explained in Zarco-Tejada et al. (in press, b).

\section{RESULTS}

Results of the leaf-level experiments and CASI canopy reflectance measurements in the laboratory are reported in detail in Zarco-Tejada et al. (in press, a, b). A typical pair of reflectance spectra obtained with the Schott filter measurement protocol is shown in Figure 1 illustrating the additive effect of the broad $740 \mathrm{~nm}$ fluorescence signal superimposed on the reflectance spectrum due only to the scattering and absorption effects within the leaf. Results of the time-decay experiment showed that changes in $\mathrm{CF}$ amplitude subsequent to exposure were also tracked in apparent reflectance spectra. Measurements taken every 2 seconds during five minutes of illumination allowed comparison between the first and last spectral reflectance measurement (Figure 2). The differences in apparent reflectance are seen at approximately $690 \mathrm{~nm}$ and $750 \mathrm{~nm}$ corresponding to the two chlorophyll fluorescence emission peaks and, in addition, a fluorescence emission of unknown origin is observed near $370 \mathrm{~nm}$ in the blue. Reflectance differences in the red edge were fitted to a double gaussian function as suggested in Subhash and Mohanan (1997) and as used in the FRT radiative transfer model for CF simulation on apparent reflectance (Zarco-Tejada et al., in press, a). Parameters showing the centre maxima and bandwidth for both fluorescence emissions from Acer saccharum M. leaf samples are shown in Table 1 and Figure 3. These fits produce high correlation coefficients $(\mathrm{R}>0.9)$, generate parameters similar to those of Subhash and Mohanan (1997) and also show bandwidths at $750 \mathrm{~nm}$ that are approximately 2 to 3 times larger than near $690 \mathrm{~nm}$.. The centre peaks show shifts from those reported earlier, but these results correspond to natural fluorescence from broadband illumination rather than from laser excitation energy in the earlier results.

Measurements of leaf reflectance collected in the diurnal trial showed the diurnal variation of fluorescence features Fv/Fm and Ft110 (Zarco-Tejada et al., in press, a), observed during the day compared to the variation of the reflectance difference at $740 \mathrm{~nm}$ with and without the blocking filter, therefore tracking the PSII excitation to visible light

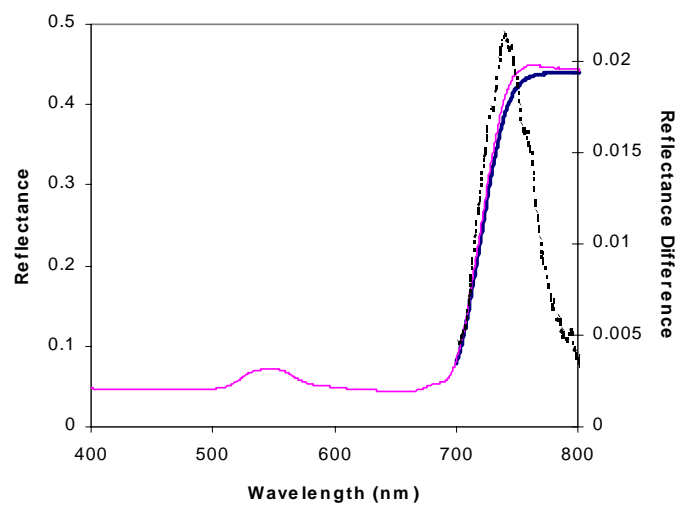

With RG695 filter — No filter ........ Difference

Figure 1. Single leaf reflectance measurements obtained with the Li-Cor 1800 apparatus and fibre spectrometer using the measurement protocol with the RG695 filter (thick line) and with no filter (thin line) from a dark-adapted Acer saccharum M. leaf sample (adapted from Zarco-Tejada et al. (in press, a).

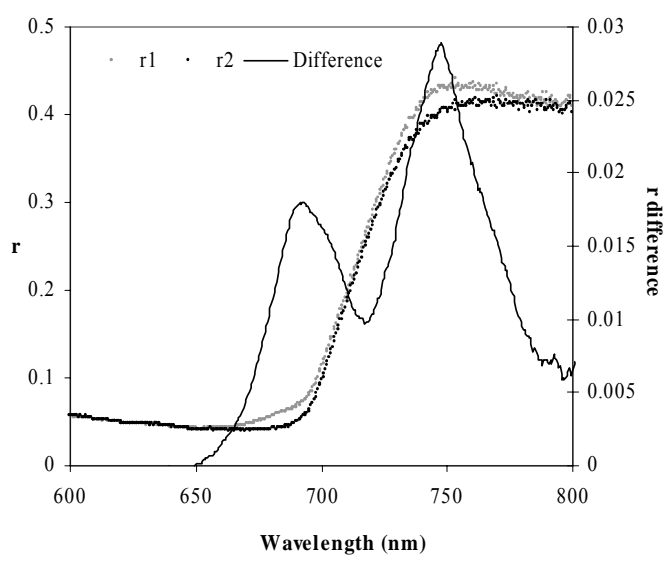

Figure 2. Reflectance measurements taken at $\mathrm{t}_{0}$ $\left(\mathrm{r}_{1}\right)$ and $\mathrm{t}_{1}(5 \mathrm{~min})\left(\mathrm{r}_{2}\right)$ in a time-decay experiment which demonstrates the effect of fluorescence emission bands on the reflectance spectra in the $600-800 \mathrm{~nm}$ spectral region due to PSII and PSI photosystems. 
superimposed on the reflectance when there is no excitation. Results showed that variations in Fv/Fm during the day are captured in the leaf reflectance measurements even when the pigment concentration is constant. CASI canopy reflectance measurements in the laboratory showed that changes in canopy apparent reflectance from targeted plant material are observed when the Schott $695 \mathrm{~nm}$ blocking filter is used. This effect is evident at $730-750 \mathrm{~nm}$, and is most pronounced at $742 \mathrm{~nm}$. When the maple-seedling canopy was kept in a fixed position during 3 minutes of CASI data acquisition in the 72-channel (7.5 $\mathrm{nm}$ bandwidth) mode of operation, changes in the CASI reflectance bands affected by chlorophyll fluorescence in this time-decay experiment were seen (Figure 4).

Table 1. The reflectance difference spectra have been fit with a double gaussian curve defined above. The best fit parameters show spectral peaks and half-widths consistent with fluorescence emissions.

Double Gaussian Fit:

$R=A 1 \cdot \exp \left(\frac{-\left(\lambda-\lambda_{1}\right)^{2}}{0.36 \cdot \Delta_{\lambda 1}^{2}}\right)+A 2 \cdot \exp \left(\frac{-\left(\lambda-\lambda_{2}\right)^{2}}{0.36 \cdot \Delta_{\lambda 2}^{2}}\right)$

\begin{tabular}{ccc} 
Param eters & Leaf_Refl & Canopy_Refl \\
\hline $\mathrm{A} 1$ & 1.7 & 1.1 \\
$\mathrm{~A} 2$ & 2.5 & 2.9 \\
$\lambda 1$ & $688.3 \mathrm{~nm}$ & $682.1 \mathrm{~nm}$ \\
$\Delta \lambda 1$ & $29.5 \mathrm{~nm}$ & $22.5 \mathrm{~nm}$ \\
$\lambda 2$ & $746.1 \mathrm{~nm}$ & $752.2 \mathrm{~nm}$ \\
$\Delta \lambda 2$ & $52.9 \mathrm{~nm}$ & $81.1 \mathrm{~nm}$ \\
$\mathrm{R}$ & 0.94 & 0.93
\end{tabular}

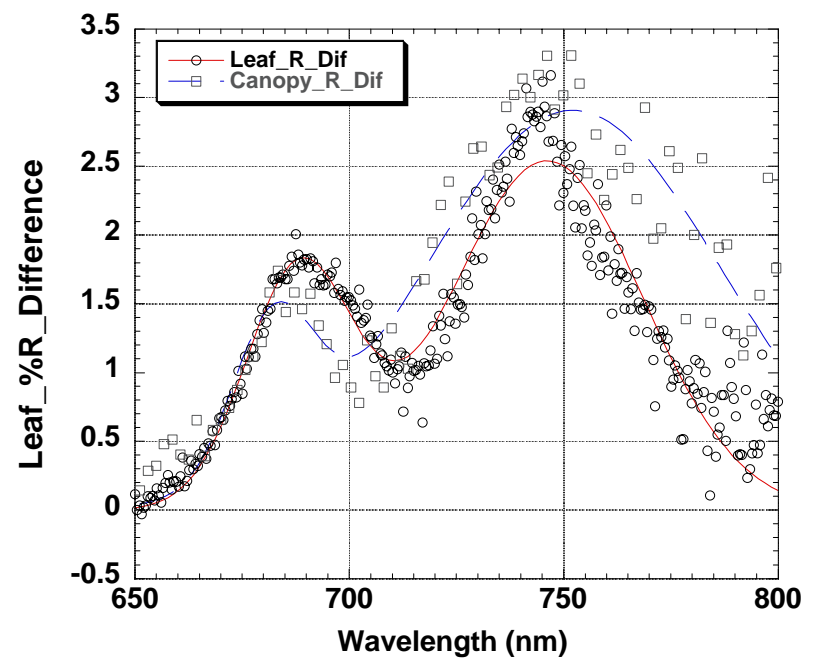

Figure 3. The measured difference reflectance spectra corresponding to Figures 2 and 4 have been fitted with a doublegaussian function. The fit parameters are presented in Table 1.

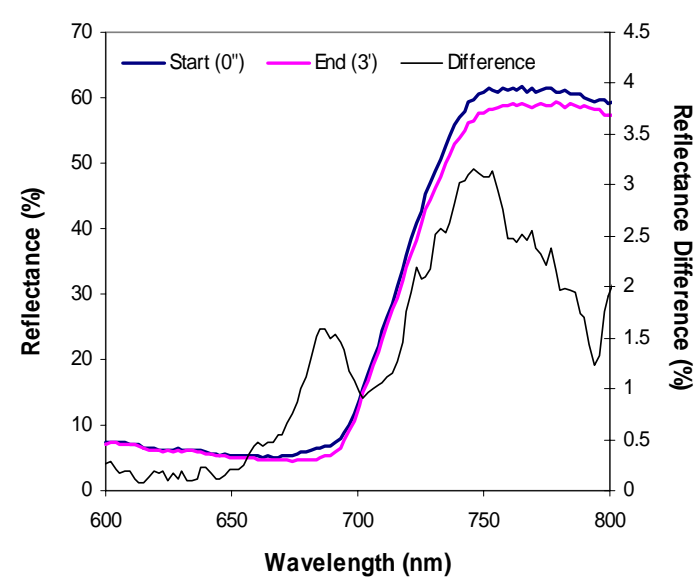

Figure 4. Effect of the CF variation in a timedecay experiment in the $600-800 \mathrm{~nm}$ canopy reflectance. CASI canopy reflectance measurements were made from Acer saccharum M. seedlings in the laboratory taken after dark adaptation and after 3 minutes of illumination. Differences in reflectances at 680-690 $\mathrm{nm}$ and 730-750 $\mathrm{nm}$ are observed due to changes in chlorophyll fluorescence.

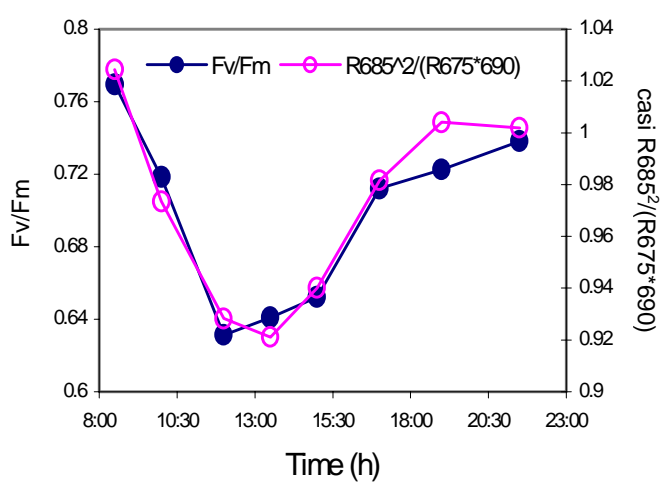

Figure 5. Diurnal variations of Fv/Fm and the optical index R685\%/(R675.690) calculated from CASI canopy reflectance in laboratory using Acer sacharum $\mathrm{M}$. seedlings. The behaviour of CF during the day is tracked by the optical index derived from CASI reflectance achieving $r^{2}=0.95$. Maple seedlings were dark-adapted for 15 minutes prior to readings of $\mathrm{Fv} / \mathrm{Fm}$ (adapted from Zarco-Tejada et al., in press, a). 
Results in the diurnal study using the CASI sensor in the laboratory with canopy seedlings showed that optical indices in the 680-690 $\mathrm{nm}$ region track changes in $\mathrm{FV} / \mathrm{Fm}$; R680/R630, R685/R630, R687/R630 and R690/R630, achieve correlation coefficients $r^{2}=0.93, r^{2}=0.94, r^{2}=0.92$, and $r^{2}=0.91$, respectively. Indices sensitive to changes in the reflectance curvature in the 675-690 nm region (ZarcoTejada et al., in press, a, b), also showed correlation with diurnal changes in $\mathrm{Fv} / \mathrm{Fm}$, i.e. curvature index $\mathrm{R}^{2} 85^{2} /(\mathrm{R} 675 \cdot 690)$ (Figure 5) yields $\mathrm{r}^{2}=0.95$.

\subsection{Above-Canopy Data Collection Experimental Results Under Natural Illumination Conditions}

Effects of CF on apparent reflectance were found in canopy reflectance measurements with a fibre spectrometer. Results of the diurnal experiments over a canopy of Acer Saccharum M. seedlings (Figure 6, upper plot), shows that reflectance difference between $8.20 \mathrm{~h}$ and $13.30 \mathrm{~h}$ is higher than reflectance difference between $8.20 \mathrm{~h}$ and 9.00am, consistent with previous diurnal experiments. The maximum in the $690-700 \mathrm{~nm}$ region due to the effects of $\mathrm{CF}$ is higher in the early morning than in mid-day, when light saturation reduces the $\mathrm{CF}$, and therefore its effects on the apparent reflectance. Dark-adapted CF measurements from the maple seedlings showed a variation in $\mathrm{Fv} / \mathrm{Fm}$ and $\mathrm{Ft}$ during the day, decreasing in the afternoon: $\quad \mathrm{Fv} / \mathrm{Fm}=0.574, \quad \mathrm{Ft}=0.495 \quad$ at $9: 09 \mathrm{~h}$; $\mathrm{Fv} / \mathrm{Fm}=0.524, \mathrm{Ft}=0.418$ at $11: 22 \mathrm{~h}$; and $\mathrm{Fv} / \mathrm{Fm}=0.516$, $\mathrm{Ft}=0.396$ at $13: 23 \mathrm{~h}$. These results demonstrate consistency with artificial light and laboratory experiments, showing that $\mathrm{CF}$ changes can be tracked under natural illumination conditions using a fibre spectrometer over small canopies. Subsequent experiments with the airborne CASI hyperspectral sensor over forest sites in 1999 and 2000 revealed promising but less convincing results. Analysis results from one of the healthy forest sites are shown in Figure 6 (lower plot) where reflectance in the 72-channel CASI mode and CF measurements were collected at different times of the day. Reflectance difference between CASI measurements at $8.00 \mathrm{~h}$ and $12.20 \mathrm{~h}$ shows a maximum at $700 \mathrm{~nm}$, consistent with higher value of reflectance at $700 \mathrm{~nm}$ due to the higher $\mathrm{CF}$ effects in the early morning before photosystem saturation. Ground truth $\mathrm{CF}$ measurements showed a decrease in the non-dark-adapted $\mathrm{Ft}$ : $\mathrm{Ft}=0.74$ at $8.00 \mathrm{~h}, \mathrm{Ft}=0.72$ at $9.30 \mathrm{~h}, \mathrm{Ft}=0.46$ at 12.30h, and dark-adapted Fv/Fm: Fv/Fm: Fv/Fm=0.83 at 8.00h, Fv/Fm=0.82 at 9.30h, Fv/Fm=0.79 at $12.30 \mathrm{~h}$. The shift in the maximum of the reflectance difference from $695 \mathrm{~nm}$ at leaf level to $700 \mathrm{~nm}$ at CASI canopy level is under investigation. Results from the experiments in June 2000 using higher spatial resolution of $0.56 \times 1.08 \mathrm{~m}$ and a CASI bandset of 9 bands centered in the $695 \mathrm{~nm}$ spectral region did not show clear and definitive results. The effect of the atmosphere with the water vapour and $\mathrm{O}_{2}$ bands in the $690-700 \mathrm{~nm}$ region was an issue investigated for both cases in which different sensors and data correction was carried out. For the measurements with the fibre spectrometer, a Spectalon panel measurement collected every time facilitated the elimination of atmospheric features in the reflectance data. For the CASI data collected at different times, a post-processing refinement after atmospheric correction was performed in order to remove residual correction errors in the 670-710 $\mathrm{nm}$ region by selecting road spectra from the images. Correction factors were applied to the data using a flat field approach, removing residual atmospheric effects in the difference spectra. Correlation coefficients were calculated from the estimation of Fv/Fm when leaf-level relationships between CF and optical indices are applied to the 12 sites of Acer saccharum M. collected with CASI in 1998 and 1999 through SAIL and Kuusk CR models (Zarco-Tejada, in press, a, b). Results show that the indices producing the best estimations of CF were the Curvature index, derivative indices such as DP22, DPR1, DPR2, and DP21 (Zarco-Tejada et al., 1999a), and indices in the visible such as PRI3 $\left(\mathrm{R}_{570}-\mathrm{R}_{539}\right) /\left(\mathrm{R}_{570}+\mathrm{R}_{539}\right)$, and $\mathrm{R}_{440} / \mathrm{R}_{690}$. 


\section{CONCLUSIONS}

This paper shows results obtained at different levels of study which demonstrate that CF signature affects the reflectance measurements acquired at leaf and canopy levels. Experiments presented here focussed on the study of the effect of CF on the canopy reflectance signature under natural illumination conditions, as a continuation of previous work carried out in the laboratory and through modelling using a radiative transfer simulation and the CASI hyperspectral sensor. Diurnal trials using a fibre spectrometer over a canopy of seedlings produced positive results, showing that canopy reflectance is affected by diurnal variation of $\mathrm{CF}$, and that such reflectance changes are measurable under natural illumination conditions. However, airborne experiments with the CASI hyperspectral sensor produced promising but inconclusive results in two diurnal experiments carried out in 1999 and 2000, in which small variations of reflectance due to the effect of CF were observed. The real applicability of these results in vegetation canopies is still under investigation due to the difficulties associated with the small changes in reflectance due to CF and the spectral regions where they occur, requiring a very accurate atmospheric correction.

\section{ACKNOWLEDGEMENTS}

Research support for this work is gratefully acknowledged from GEOIDE, a Canadian Networked Centre of Excellence, and the Centre for Research in Earth and Space Technology.

\section{REFERENCES}

Heinz-Walz-GmbH, 1993. Portable Fluorometer PAM-2000 and data acquisition software DA-2000, Effeltrich, Germany.

Mohammed, G. H., Binder, W. D., and Gillies, S. L., 1995. Chlorophyll fluorescence: A review of its practical forestry applications and instrumentation. Scand. J. For. Res. 10:383-410.

Mohammed, G. H., Sampson, P. H., Colombo, S. J., Noland, T. L., and Miller, J. R., 1997. Bioindicators of forest sustainability: Development of a forest condition rating system for Ontario project strategy, Report 137, Ontario Forest Research Institute, Sault Ste. Marie, ON.

Sampson, P. H., Mohammed, G. H., Colombo, S. J., Noland, T. L., Miller, J. R., Zarco-Tejada, P. J., 1998. Bioindicators of forest sustainability progress report, Report 142, Ontario Forest Research Institute, Sault Ste. Marie, ON.

Subhash, N. and Mohanan, C. N., 1997. Curve-fit analysis of chlorophyll fluorescence spectra: Application to nutrient stress detection in sunflower. Remote Sens. Environ. 60:347-356.

Zarco-Tejada, P. J., Miller, J. R., Mohammed, G. H., Noland, T. L. and Sampson, P. H., 1999a. Canopy Optical Indices from Infinite Reflectance and Canopy Reflectance Models for Forest Condition Monitoring: Application to Hyperspectral CASI data, In IEEE 1999 International Geoscience and Remote Sensing Symposium, IGARSS'99, Hamburg (Germany).

Zarco-Tejada, P. J., Miller, J. R., Mohammed, G. H., Noland, T. L. and Sampson, P. H., 1999b. Optical Indices as Bioindicators of Forest Condition from Hyperspectral CASI data, In Proceedings 19th Symposium of the European Association of Remote Sensing Laboratories (EARSeL), Valladolid (Spain)

Zarco-Tejada, P. J., Miller, J. R., Mohammed, G. H., Noland, T. L., (in press, a). Chlorophyll Fluorescence Effects on Vegetation Apparent Reflectance: I. Leaf-Level Measurements and Model Simulation, Remote Sensing of Environment.

Zarco-Tejada, P. J., Miller, J. R., Mohammed, G. H., Noland, T. L and Sampson, P.H., (in press, b). Chlorophyll Fluorescence Effects on Vegetation Apparent Reflectance: II. Laboratory and Airborne Canopy-Level Measurements with Hyperspectral data, Remote Sensing of Environment. 